\title{
OUTCOME OF LAPAROSCOPIC SLEEVE GASTRECTOMY AND LAPAROSCOPIC MINI GASTRIC BYPASS ON EGYPTIAN MORBID OBESE PATIENTS
}

\author{
Osama Ali ElAtrash, Walid Ibrahim Abdel Hamid, Mohamed Abdel Monem \\ Marzouk, Mohamed Abdel Satar Abdel Hamid, and Ahmed Farouk Abdel Hafeez
}

\author{
Department of General Surgery, \\ Faculty of medicine, Ain Shams \\ University. Cairo, Egypt

\section{Corresponding:} \\ Ahmed Farouk Abdel Hafeez \\ Mobile : 01005592127 ; \\ E mail: \\ dr.ahmedzorro@gmail.com \\ Received: 10/2/2020 \\ Accepted: 2/3/2020
}

Online ISSN: 2735-3540

\begin{abstract}
Background: Chronic diseases are well established as the predominant death cause, and obesity, being one of the factors strongly contributive to chronic diseases, has been consistently threatening the global health.
\end{abstract}

Aim of the Work: To compare between laparoscopic mini gastric bypass and laparoscopic sleeve gastrectomy on morbid obese patients in Egypt as regard weight loss, outcome of associated co-morbiditis and with 1 year follow up after operation.

Patients and Methods: This study included 200 patients, 100 patients in each group. The group age ranged between $22-55$ years with a mean $\pm S D$ of $37.88 \pm 9.52$ years. Female patients represented the main population of this study (60.5\%). It is a common finding in the literature that women are undergoing bariatric surgery more than men. We included in this study patients with BMI $\geq 40$ or BMI $=35-39$ with one or more obesity-related co morbidities. It is a sample study which was done between December 2016 - December 2018 at Ain Shams University Hospitals, Cairo, Egypt.

Results: Finally our study suggests that bariatric surgery (mainly $L S G$ and LMGB which are the most common bariatric operations nowadays) are considered the best treatment of diabetes type 2 in obese patients and LMGB is superior to LSG in diabetes remission. Both procedures are also associated with improvement of the blood pressure and the sleep apnea owing to weight loss.

Conclusion: It is reasonable to say that both LSG and LMGB achieve similar weight loss mean and resolution of co-morbidities at 1 year.

Keywords: Haemoglobin Al c, Laparoscopic mini gastric bypass, Laparoscopic sleeve gastrectomy, American diabetes association

\section{INTRODUCTION:}

Obesity is a chronic disease that impairs health-related quality of life in adolescents and children. In 2010, overweight and obesity were estimated to cause 3.4 million deaths, $3.9 \%$ of years of life loss, and 3.8\% of disability-adjusted life-years worldwide (1).
Weight loss surgery has become increasingly recognized as effective treatment for these comorbidities, and it is considered to be a reasonable option when non surgical methods of weight loss fail. Current studies suggest that neither pharmacologic nor dietary treatment can maintain weight loss in obese patients as effectively as can weight loss surgery ${ }^{(2)}$. 
Sleeve gastrectomy is a bariatric technique consisting of subtotal vertical gastrectomy with preservation of the pylorus, including longitudinal resection of fundus, corpus and antrum, to create a tubular duct along the lesser curvature. Resection comprises approximately $80 \%$ of the stomach and the remnant gastric has a capacity $>100 \mathrm{~mL}$. It is considered an easier technique than other procedures ${ }^{(3)}$.

Laparoscopic mini-gastric bypass is reported to be a safe alternative to LRYGB, showing comparable efficacy in weight reduction and resolution of metabolic complications, including diabetes ${ }^{(4)}$.

\section{AIM OF THE WORK:}

The aim of this randomized trial study to compare between laparoscopic mini gastric bypass and laparoscopic sleeve gastrectomy on morbid obese patients in Egypt as regard weight loss, outcome of associated co-morbiditis and with 1 year follow up after operation.

\section{PATIENTS AND METHODS:}

Study Design: It is a sample study which was done between December 2016 December 2018 at Ain Shams university hospitals, Cairo, Egypt.

Study Population: This study included 200 morbidly obese patients withdivided into two groups: Group (1): (100 patients) treated by laparoscopic Sleeve gastrectomy. Group (2): (100 patients) treated by laparoscopic Mini-Gastric Bypass.

All patients were operated with the same surgical team in Ain Shams University Hospitals.

\section{Inclusion criteria:}

The patients included in this study fulfilled the following criteria: They were willing to give consent and comply with the evaluation and treatment schedule. (The patients treated by LSG refused to take multivitamins for life and the patients treated by MGB were sweet addicts). Their age between 18 and 60 years. Their body mass index (BMI) $\geq 35 \mathrm{~kg} / \mathrm{m} 2$. Supportive family/ social environment. No alchol or substance abuse. Absence of active untreated depression or schizophrenia.

\section{Exclusion criteria:}

The patients who were excluded from the study: Endocrine abnormalities: e.g. hypothyroidism, Cushing syndrome. Previous bariatric operations. Major upper abdominal surgery. Age below 18 years old or more than 60 years. Pregnant or lactating females. Patient with contraindications for insufflation as those with sever cardiovascular or sever restrictive respiratory diseases. Patient with significant abdominal ventral hernia. Patient with major psychiatric illness.

\section{Preoperative workup:}

All patients were subjected to the following:

Complete history taking: Personal history: as age, sex, marital status. Feeding history and if the patients likes sweet much or not. Duration of obesity. History of previous trials of weight loss whether surgical or non-surgical. Medical history for co morbidities: DM: type, onset, course, duration, current medications, controlled or not, if change from oral hypoglycemic to insulin and when, family history. Hypertension. Cardiac and respiratory problems. Sleep symptoms questionnaire. Family history of obesity. Previous DVT. Any other morbidity. Past surgical history.

\section{Complete physical examination:} Measurement of weight per $\mathrm{Kg}$, height per meter then calculation of BMI = (weight $\mathrm{Kg} /$ height $\mathrm{m}^{2}$ ). Abdominal examination for (scar for pervious surgery, hernia orifices, organomegaly, right hypochondrial tenderness). Cardiac and pulmonary 
evaluation. Medical consultation for proper control of blood sugar and hypertension.

\section{Investigations:}

Laboratory investigation: CBC,LFT, KFT, FBS, 2hours Postprandial blood sugar, serum insulin level, C-peptide level, $\mathrm{HbAlc}$, coagulation profile, serum Calcium, $\mathrm{Na}, \mathrm{K}$, $\mathrm{Mg}$, thyroid function tests, serum cortisol level, lipid Profile.

Other investigation: Chest X-ray, abdominal U.S, pulmonary function test, echocardiography, UGI endoscopy (if needed).

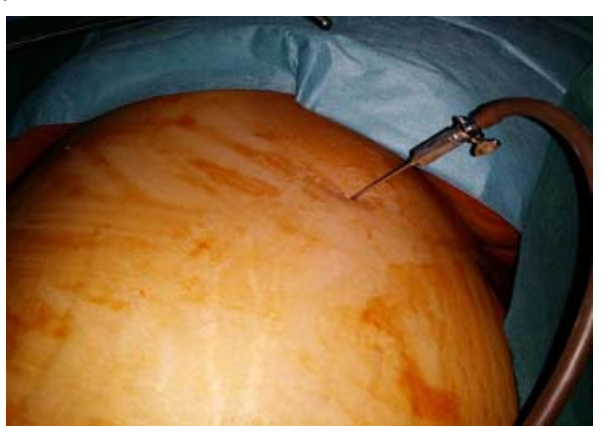

\section{Operative Techniques:}

The first group of sleeve gastrectomy: technique was done by laparoscopy with the following steps: the patient is positioned in reverse Trendelenburg position with splitting of the legs (French position) and abducted arms. $\mathrm{CO}_{2}$ insufflation is done through a Verress needle placed in left subcostal region at midclavicular line. Five ports are inserted, a 5-12-mm port is placed under direct vision approximately $15 \mathrm{~cm}$ below the xiphoid and $3 \mathrm{~cm}$ to the left of midline (Figure 1).

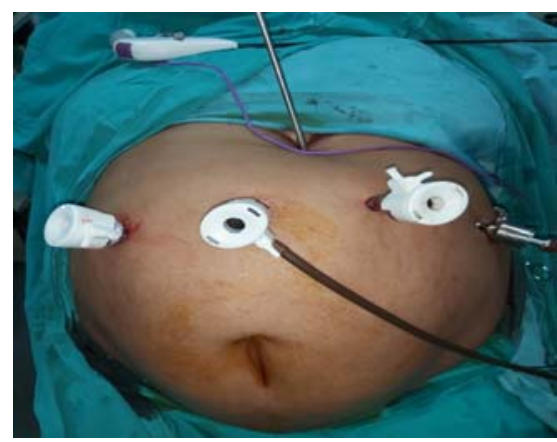

Figure (1): Five ports insertion.

A 30-degree angled laparoscope is placed through the port into the peritoneal cavity and 5-12-mm port is placed in the left lateral flank (right hand of surgeon), is placed at the level of the left midclavicular line with the patient in a supine position and at the same level as the periumbilical port. Next, a 5-mm trocar port is placed along the left subcostal margin between the xiphoid process and the left flank port in the left anterior axillary line (grasper of assistant). Another 12-15-mm port is placed in the right upper quadrant region at midclavicular line (left hand of surgeon) and a $5 \mathrm{~mm}$ port was placed in the mid-epigastric region for retraction of the left liver lobe by Nathanson liver retractor.
The pylorus of the stomach is then identified and the greater curve of the stomach is elevated (Figure 2). A laparoscopic harmonic $\AA$ scalpel (or Ligasure $\AA$ ) is then used to enter the greater sac via division of the greater omentum (Figure 3). The greater curvature of the stomach is then dissected free from the omentum starting $2-4 \mathrm{~cm}$ from the pylorus and proceeds to the short gastric blood vessels (taking care to avoid injury of spleen) and angle of Hiss (Figure 4\&5). The left crus is completely freed from any attachment to avoid leaving any posterior pouch or fundus remnant and to be sure that there is no hiatus hernia. (Figure 6). Then freeing of any posterior attachments between stomach and pancreas (posterior gastric adhesions) (Figure 7). 


\section{Osama Ali ElAtrash, et al.,}

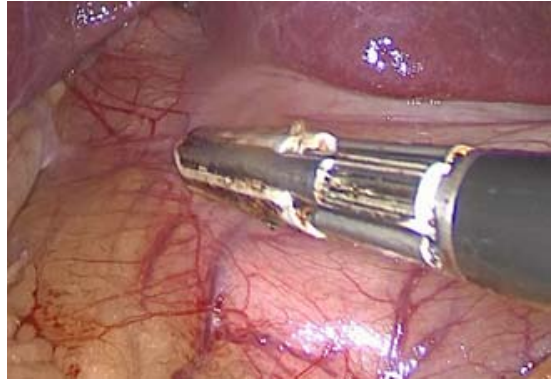

Figure (2): Identification of pyloric ring.

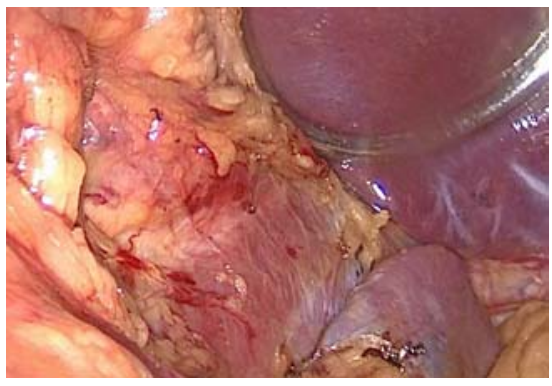

Figure (5): Complete freeing of left crus.

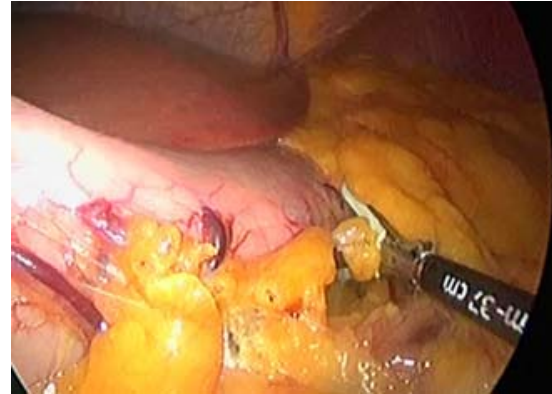

Figure (3): Opening of greater omentum.

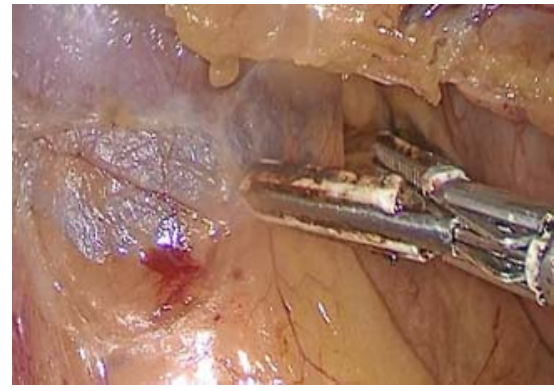

Figure (6): Dissection of posterior gastric wall from pancreas.

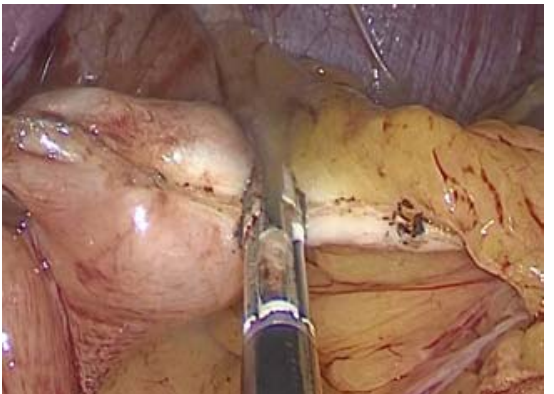

Figure (4): Dissection of greater omentum.
A 36 French bougie is used as a template to perform the vertical sleeve gastrectomy beginning 2-4 $\mathrm{cm}$ proximal to the pylorus and extending to the angle of Hiss by a $60-\mathrm{mm}$ stapler along and guided by the bougie, the first used stapler is green cartridge (due to more thick antral stomach) and the remaining staplers are blue cartridge stapler. Then vertical gastric pouch is completely separated from the small tubular (sleeve like) stomach pouch.

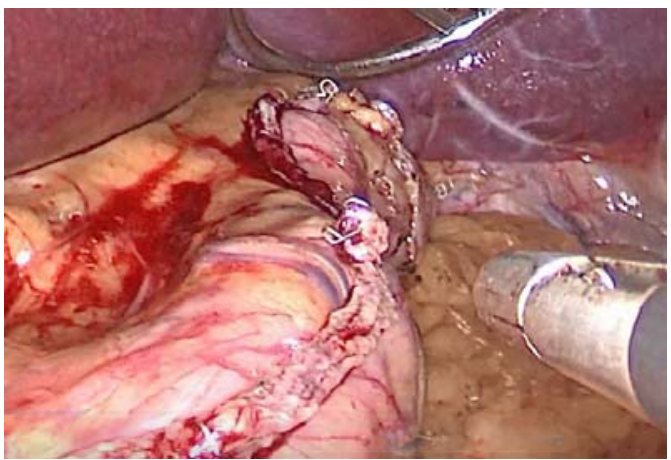

Figure (7): The remaining sleeve like gastric pouch after completion of transection.

The staple line along the remaining tubularized stomach is then tested for any leak through methylene blue test (Figure 8). The staple line is also evaluated for bleeding. The gastric suture line was not systematically reinforced except in the case of bleeding and fixation of sleeve with interrupted PDS ${ }^{\circledR}$ or Vicryl sutures to omentum or pre pancreatic fascia if needed, in which case a drain was placed along the staple line. 


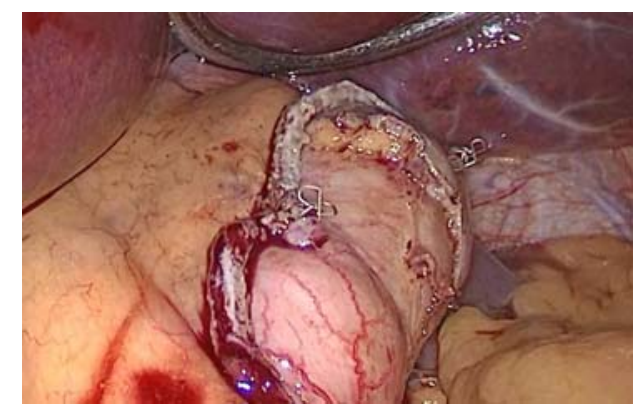

Figure (8): Gastric pouch is distended with methylene blue to test leak.

The second group of minigastric bypass: technique was done by laparoscopy with the following steps the same first group: Dissection of phrenogastric ligament (Figure 9). A window is created to enter the lesser sac between the vagus nerve and the

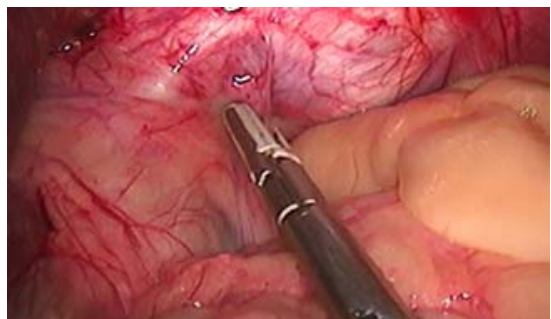

Figure (9): Dissection of Phrenogastric ligament.

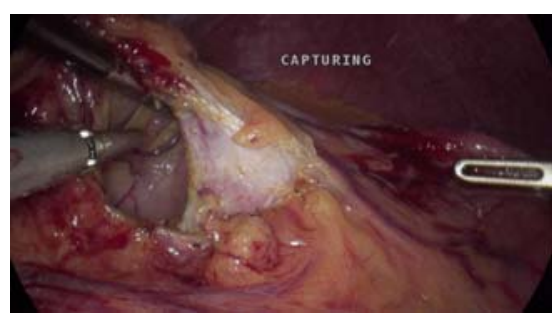

Figure (10): Opening window in lesser omentum.

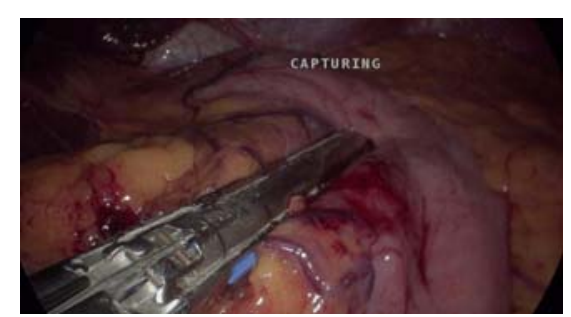

Figure (11): First stapler passing horizontally to the stomach axis.
Anterior gastrostomy is done in the new pouch. After elevation of the transverse colon and the transverse meso-colon, the ligament of Trietz was identified. Measurement of $200 \mathrm{~cm}$ of jejunum from the ligament of Treitz, Then, we approximated the bowel loop to the gastric pouch,when both are in position, the Hook were used to make an opening in the small bowel but the distal small intestines are assessed to be at least 2 miters. An antecolic-antegastricterminolateral gastrojejunostomy is performed using $60 \mathrm{~mm}$ blue stapler (Figure 12).

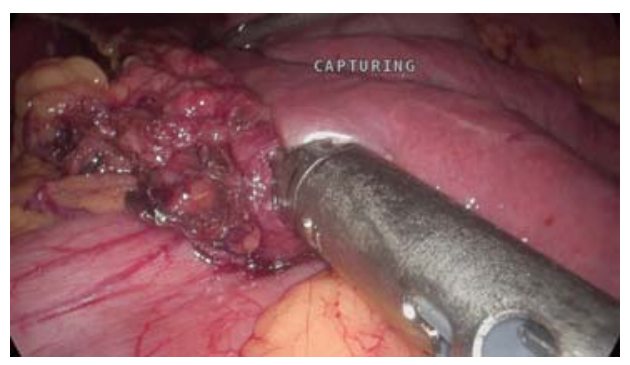

Figure (12): Stapler gastrojejunostomy between the gastric pouch and jejunal loop $200 \mathrm{~cm}$ from Treitz's ligament.

Then the stoma opening was closed with two layers continuous sutures using 2/0 absorbable V-lock ${ }^{\circledR}$ over a Ryle tube inserted through the oral cavity and carefully introduced through the stoma opening to efferent intestinal loop (Figure 13\&14). Thereafter, leak test was performed through injection of about $50 \mathrm{cc}$ of Methylene blue dye 
while both afferent and efferent loops were closed by intestinal clamps, anastomosis was carefully inspected all through and should be water tight (Figure 15).

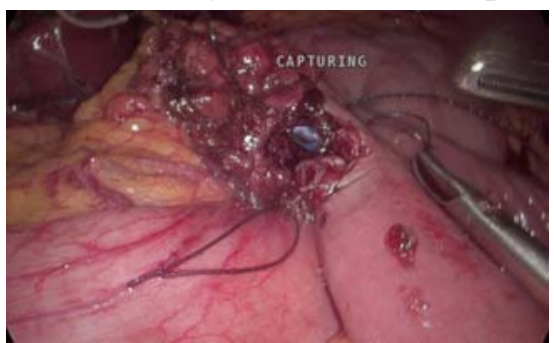

Figure (13): Passing rhyle tube through the anastomosis opening

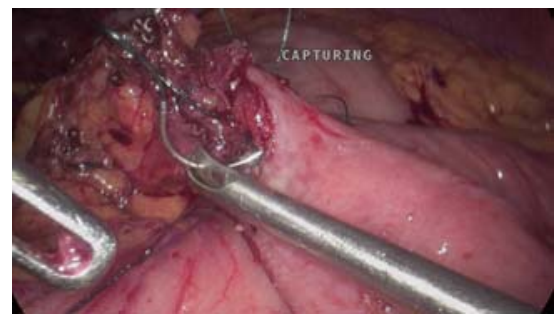

Figure (14): Closure of the residual stoma by Vicryl Sutures.

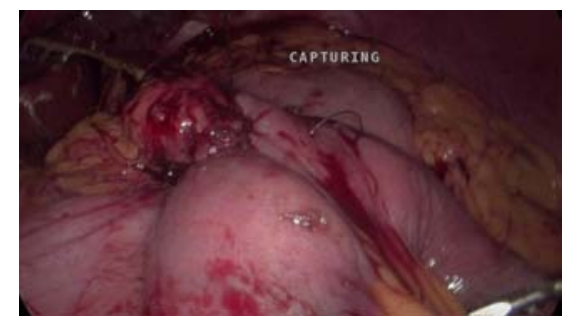

Figure (15): Methylene blue test
Postoperative care: Close observation for vital signs (ICU admission if indicated). Chest physiotherapy. Encourage early mobilization. Low molecular weight heparin during hospital stay. The patients received proton pump inhibitors to avoid stress ulcers. One dose of one gram intravenous third generation cephalosporin. Proper pain management. Oral clear fluid started one day after surgery and maintained for two weeks. The Drain is usually removed before discharge in LSG group and left for 5-7 days in MGB group.

Outcomes Assessment: Operative time. Hospital stay. Rate of conversion. Time to oral feeding. Weight loss depending on the change in BMI which was measured at the initial screening on the day of surgery, 1 week at stitch removal and at 1, 3, 6, and 12 months after surgery. Intraoperative and postoperative complications (early or late) were recorded for each operations D.M control by measurement of $\mathrm{HbAlc}$ at 3,6 and 12 months and FBS at 1,3, 6 and 12 months with follow up of changes in dose or discontinuation of anti-diabetic medications Hypertension control by measurement of Blood pressure at 1, 3, 6 and 12 months with follow up of changes or discontinuation of Antihypertensive medications. Sleep apnea syndrome follow up for patients with past history of it using sleep symptoms questionnaire.

\section{Data management and statistical analysis:}

The collected data was revised, coded, tabulated and introduced to a PC using Statistical package for Social Science (IBM Corp. Released 2011. IBM SPSS Statistics for Windows, Version 20.0. Armonk, NY: IBM Corp). Data was presented and suitable analysis was done according to the type of data obtained for each parameter:

Descriptive statistics: Mean, Standard deviation $( \pm$ SD) and range for parametric numerical data, Frequency and percentage of non-numerical data.

Analytical statistics: Student $\mathrm{T}$ Test was used to assess the statistical significance of the difference between two study group means. Chi-Square test was used to examine the relationship between two qualitative variables. Fisher's exact test: was used to examine the relationship between two qualitative variables when the expected count is less than 5 in more than $20 \%$ of cells. Logistic regression: used in the prediction of the presence or absence of an outcome based on a set of independent variables.

P- value: level of significance: $\mathrm{P}>0.05$ : Non significant (NS). $\mathrm{P}<0.05$ : Significant (S). $\mathrm{P}<0.01$ : Highly significant (HS). 


\section{RESULTS}

Table (1): Comparison between group 1 (sleeve gastrectomy) and group 2 (minigastric bypass) as regard personal characteristics.

\begin{tabular}{|c|c|c|c|c|c|c|c|}
\hline & \multicolumn{4}{|c|}{ Group } & \multirow{3}{*}{$P$ value } & \multirow{3}{*}{ Sig. } \\
\hline & & \multicolumn{2}{|c|}{ Sleeve gastrectomy } & \multicolumn{2}{|c|}{ Minigastric bypass } & & \\
\hline & & Mean & $\pm \mathrm{SD}$ & Mean & $\pm \mathrm{SD}$ & & \\
\hline \multicolumn{2}{|l|}{ Age } & 37.73 & 9.35 & 37.47 & 9.05 & $0.911^{*}$ & NS \\
\hline & & No. & $\%$ & No. & $\%$ & & \\
\hline \multirow[t]{2}{*}{ Sex } & Male & 37 & $37 \%$ & 42 & $42.0 \%$ & \multirow[t]{2}{*}{$0.592 * *$} & \multirow[t]{2}{*}{ NS } \\
\hline & Female & 63 & $63 \%$ & 58 & $58.0 \%$ & & \\
\hline \multirow{2}{*}{$\begin{array}{l}\text { Family history of } \\
\text { DM }\end{array}$} & Negative & 12 & $40.0 \%$ & 19 & $21 \%$ & \multirow[t]{2}{*}{$0.273^{* *}$} & \multirow[t]{2}{*}{ NS } \\
\hline & Positive & 18 & $60.0 \%$ & 70 & $79 \%$ & & \\
\hline
\end{tabular}

Table (2): Comparison between group 1 (sleeve gastrectomy) and group 2 (minigastric bypass) as regard biochemical characteristics.

\begin{tabular}{|l|c|c|c|c|c|c|}
\hline \multirow{2}{*}{} & \multicolumn{4}{|c|}{ Group } & \multirow{2}{*}{ P value } & \multirow{2}{*}{ Sig. } \\
\cline { 2 - 5 } & \multicolumn{2}{|c|}{ Sleeve gastrectomy } & \multicolumn{2}{|c|}{ Minigastric bypass } & & \\
\cline { 2 - 5 } & Mean & \pm SD & Mean & \pm SD & & \\
\hline C-peptide & 3.77 & 1.25 & 3.90 & 1.37 & $0.549^{*}$ & NS \\
\hline BMI baseline & 51.93 & 9.78 & 52.33 & 9.41 & $0.421^{*}$ & NS \\
\hline FBS baseline & 138.27 & 15.78 & 140.67 & 12.27 & $0.514^{*}$ & NS \\
\hline HbA1c baseline & 8.01 & 0.80 & 8.10 & 0.92 & $0.326^{*}$ & NS \\
\hline Systolic Bl.Pr & 145.7 & 10.7 & 143.5 & 11.7 & $0.99^{*}$ & NS \\
\hline Diastolic Bl Pr & 86.3 & 8.9 & 90.4 & 8.6 & 0.97 & NS \\
\hline
\end{tabular}

*Student $\mathrm{t}$ tests.

Table (3): Comparison between group 1 (sleeve gastrectomy) and group 2 (minigastric bypass) as regard medical characteristics.

\begin{tabular}{|c|c|c|c|c|c|c|c|}
\hline & \multicolumn{4}{|c|}{ Group } & \multirow{3}{*}{$P$ value } & \multirow{3}{*}{ Sig. } \\
\hline & & \multicolumn{2}{|c|}{$\begin{array}{c}\text { Sleeve } \\
\text { gastrectomy }\end{array}$} & \multicolumn{2}{|c|}{$\begin{array}{c}\text { Minigastric } \\
\text { Bypass }\end{array}$} & & \\
\hline & & No. & $\%$ & No. & $\%$ & & \\
\hline \multirow[t]{3}{*}{ Distribution of obesity } & Peripheral & 13 & $13 \%$ & 26 & $26 \%$ & \multirow[t]{3}{*}{$0.235^{*}$} & \multirow[t]{3}{*}{$\mathrm{NS}$} \\
\hline & Central & 34 & $34 \%$ & 40 & $40 \%$ & & \\
\hline & Both & 53 & $53 \%$ & 34 & $33 \%$ & & \\
\hline \multirow[t]{2}{*}{ D.M } & yes & 65 & $65 \%$ & 89 & $89 \%$ & \multirow[t]{2}{*}{$0.593 *$} & \multirow[t]{2}{*}{ NS } \\
\hline & no & 35 & $35 \%$ & 11 & $11 \%$ & & \\
\hline \multirow{2}{*}{$\begin{array}{l}\text { Preoperative } \\
\text { medication }\end{array}$} & OHG & 51 & $78.5 \%$ & 51 & $57 \%$ & \multirow[t]{2}{*}{$0.573^{*}$} & \multirow[t]{2}{*}{ NS } \\
\hline & Insulin & 14 & $21.5 \%$ & 38 & $43 \%$ & & \\
\hline \multirow{2}{*}{$\begin{array}{l}\text { Status of D.M } \\
\text { (according to } \\
\text { baselineHbA1c) }\end{array}$} & $\begin{array}{l}\text { Less Control } \\
>8.5 \%\end{array}$ & 12 & $18.5 \%$ & 46 & $52 \%$ & \multirow[t]{2}{*}{$1.0^{*}$} & \multirow[t]{2}{*}{$\mathrm{NS}$} \\
\hline & $\begin{array}{l}\text { Better Control } \\
<8.5 \%\end{array}$ & 53 & $81.5 \%$ & 43 & $48 \%$ & & \\
\hline \multirow[t]{2}{*}{ C-peptide } & $<3 \mathrm{ng} / \mathrm{ml}$ & 13 & $20.0 \%$ & 23 & $26 \%$ & \multirow[t]{2}{*}{$0.542 *$} & \multirow[t]{2}{*}{ NS } \\
\hline & $>3 \mathrm{ng} / \mathrm{ml}$ & 52 & $80.0 \%$ & 66 & $74 \%$ & & \\
\hline \multirow[t]{2}{*}{ Hypertension } & $\begin{array}{l}\text { Not on } \\
\text { medication }\end{array}$ & 68 & $68 \%$ & 71 & $71 \%$ & \multirow[t]{2}{*}{0.957} & \multirow[t]{2}{*}{$\mathrm{NS}$} \\
\hline & On medications & 60 & $88.2 \%$ & 49 & $69 \%$ & & \\
\hline Sleep apnea & & 73 & $73 \%$ & 69 & $69 \%$ & 1.05 & NS \\
\hline
\end{tabular}




\section{Osama Ali ElAtrash, et al.,}

Table (4): Comparison between group 1 (sleeve gastrectomy) and group 2 (minigastric bypass) as regard operative time.

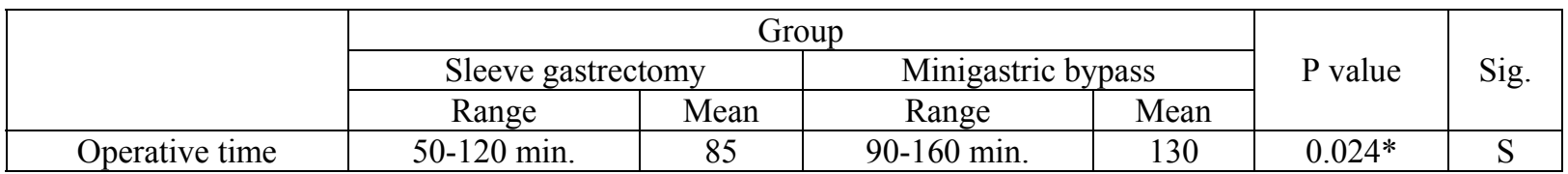

*Student $\mathrm{t}$ tests.

Table (5): Comparison between group 1 (sleeve gastrectomy) and group 2 (minigastric bypass) as regard hospital stay

\begin{tabular}{|c|c|c|c|c|c|c|}
\hline \multirow{2}{*}{} & \multicolumn{4}{|c|}{ Group } & \multirow{2}{*}{ P value } & \multirow{2}{*}{ Sig. } \\
\cline { 2 - 5 } & \multicolumn{2}{|c|}{ Sleeve gastrectomy } & Minigastric bypass & \\
\cline { 2 - 5 } & Range & Mean & Range & Mean & & \\
\hline Hospital stay & $1-3$ days. & 2 & $1-5$ days. & 3 & $0.75^{*}$ & NS \\
\hline
\end{tabular}

*Student $\mathrm{t}$ tests.

Table (6): Comparison between group 1 (sleeve gastrectomy) and group 2 (minigastric bypass) as regard complications.

\begin{tabular}{|l|c|c|c|c|}
\hline & SG group & MGB group & P value & Sig. \\
\hline Bleeding & 4 & 1 & 0.174 & NS \\
\hline Wound infection & 3 & 0 & 0.214 & NS \\
\hline Leakage & 2 & 1 & 0.560 & NS \\
\hline GIT symptoms & 5 & 7 & 0.551 & NS \\
\hline Malnutrition & 0 & 1 & 0.156 & NS \\
\hline Cholelithiasis & 1 & 3 & 0.312 & NS \\
\hline Mortality & 0 & 0 & - & - \\
\hline
\end{tabular}

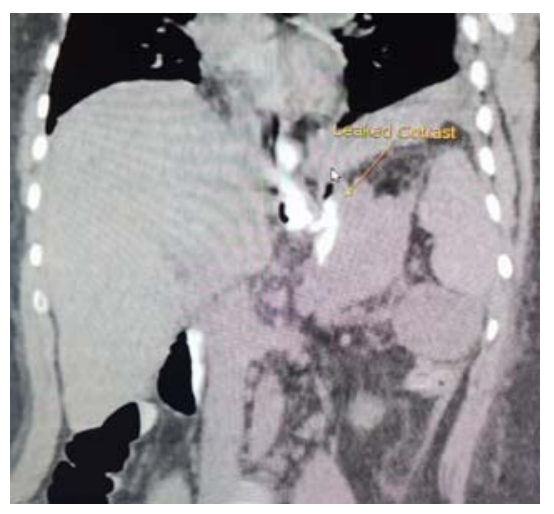

Figure (16): CTPA for a case of leakage post LSG.
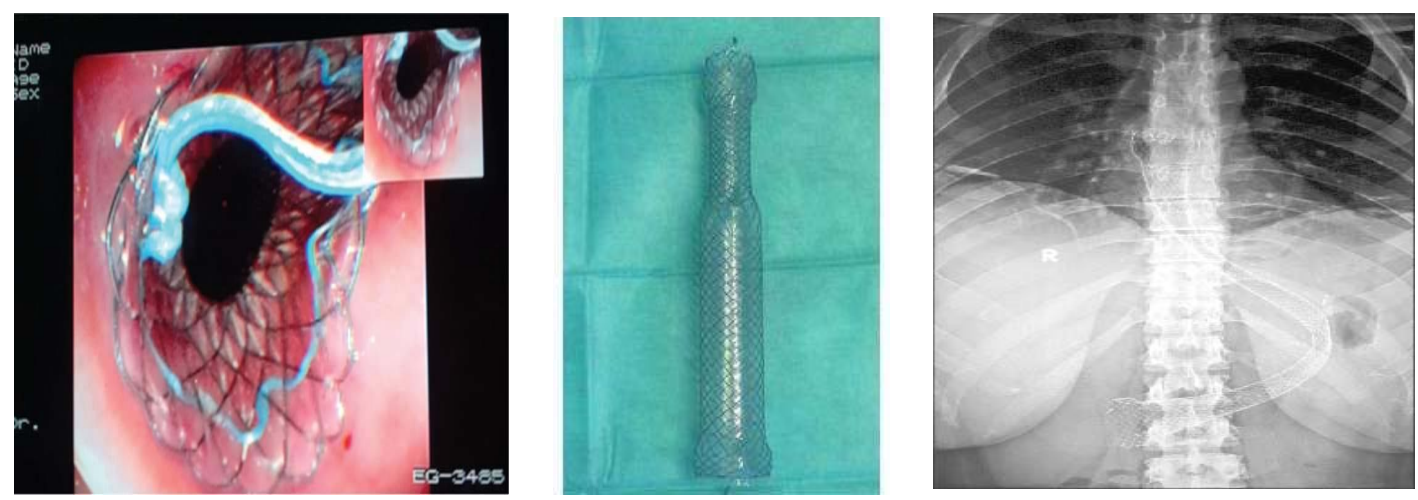

Figure (17): Mega stent insertion via upper GIT endoscopy in a case of leakage post LSG. 


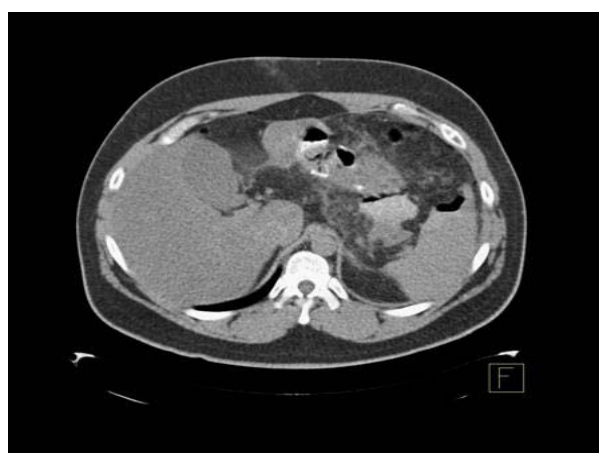

Figure (18): CTPA for a case of leakage post MGB.

Table (7): Comparison between group 1 and 2 as regard BMI at baseline, at follow up and overall BMI loss

\begin{tabular}{|c|c|c|c|c|c|c|}
\hline \multirow{2}{*}{} & \multicolumn{4}{|c|}{ Group } & \multirow{2}{*}{ P value } & \multirow{2}{*}{ Sig. } \\
\cline { 2 - 5 } & \multicolumn{2}{|c|}{ Sleevegastrectomy } & \multicolumn{2}{|c|}{ Minigastric bypass } & \\
\cline { 2 - 5 } & Mean & \pm SD & Mean & \pm SD & & NS \\
\hline BMI baseline & 51.93 & 9.78 & 51.53 & 9.41 & 0.872 & NS \\
\hline BMI 1 month & 48.2 & 8.8 & 48.3 & 8.5 & 0.924 & NS \\
\hline BMI 3 months & 44.00 & 8.49 & 43.93 & 7.94 & 0.975 & NS \\
\hline BMI 6 months & 37.73 & 6.92 & 36.73 & 4.83 & 0.519 & $\mathrm{NS}$ \\
\hline BMI12 months & 33.47 & 5.69 & 31.87 & 3.66 & 0.200 & $\mathrm{NS}$ \\
\hline Total BMI loss & 18.47 & 5.14 & 19.67 & 7.17 & 0.459 & \\
\hline
\end{tabular}

*Student $\mathrm{t}$ test

Table (8): Comparison between group 1 and 2 as regard FBS at baseline, at follow up and overall FBS change

\begin{tabular}{|l|c|c|c|c|c|c|}
\hline \multirow{2}{*}{} & \multicolumn{3}{|c|}{ Group } & \multirow{2}{*}{ P value } & \multirow{2}{*}{ Sig. } \\
\cline { 2 - 6 } & \multicolumn{2}{|c|}{ Sleeve gastrectomy } & \multicolumn{2}{|c|}{ Minigastric bypass } & \multirow{2}{*}{} \\
\cline { 2 - 6 } & Mean & \pm SD & Mean & \pm SD & & \\
\hline FBS baseline & 138.27 & 15.78 & 140.67 & 12.27 & 0.179 & NS \\
\hline FBS 1 month & 140.72 & 12.3 & 137.3 & 11.4 & 0.332 & NS \\
\hline FBS 3 months & 132.47 & 11.31 & 135.47 & 9.99 & 0.281 & NS \\
\hline FBS 6 months & 124.20 & 10.99 & 125.07 & 10.66 & 0.758 & NS \\
\hline FBS 12 months & 115.33 & 13.79 & 111.87 & 12.05 & 0.304 & NS \\
\hline Total FBS change & 29.93 & 12.84 & 37.80 & 6.41 & 0.004 & HS \\
\hline
\end{tabular}

\section{*Student $\mathrm{t}$ tests}

Table (9): Comparison between group 1 and 2 as regard HbA1c at baseline, at follow up and overall HbAlc change

\begin{tabular}{|c|c|c|c|c|c|c|}
\hline & \multicolumn{4}{|c|}{ Group } & \multirow{3}{*}{$\mathrm{P}$ value } & \multirow{3}{*}{ Sig. } \\
\hline & \multicolumn{2}{|c|}{ Sleeve gastrectomy } & \multicolumn{2}{|c|}{ Minigastric bypass } & & \\
\hline & Mean & $\pm \mathrm{SD}$ & Mean & $\pm \mathrm{SD}$ & & \\
\hline HbA1c baseline & 8.01 & 0.80 & 8.10 & 0.92 & 0.648 & $\mathrm{NS}$ \\
\hline HbAlc 3 months & 7.35 & 0.81 & 6.84 & 0.76 & 0.014 & $\mathrm{~S}$ \\
\hline HbA1c 6 months & 6.70 & 0.71 & 6.21 & 0.71 & 0.009 & $\mathrm{HS}$ \\
\hline HbA1c 12 months & 6.20 & 0.73 & 5.77 & 0.67 & 0.022 & $\mathrm{~S}$ \\
\hline Total HbAlc change & 2.01 & 0.59 & 2.33 & 0.48 & 0.024 & $\mathrm{~S}$ \\
\hline
\end{tabular}

*Student $t$ tests 
Table (10): Comparison between group 1 and 2 as regard outcome at 6 month and at final assessment diabetes.

\begin{tabular}{|c|c|c|c|c|c|c|c|}
\hline & & \multicolumn{4}{|c|}{ Group } & \multirow{3}{*}{$P$ value } & \multirow{3}{*}{ Sig. } \\
\hline & & \multicolumn{2}{|c|}{ Sleeve gastrectomy } & \multicolumn{2}{|c|}{ Minigastric bypass } & & \\
\hline & & $\mathrm{N}$ & $\%$ & $\mathrm{~N}$ & $\%$ & & \\
\hline \multirow[t]{2}{*}{ Resolved at $6 \mathrm{~ms}$} & No & 52 & $80.0 \%$ & 48 & $54 \%$ & \multirow[t]{2}{*}{$0.028 *$} & \multirow[t]{2}{*}{$\mathrm{S}$} \\
\hline & Yes & 13 & $20.0 \%$ & 41 & $46 \%$ & & \\
\hline \multirow[t]{3}{*}{ Final outcome } & No change & 5 & $7.7 \%$ & 0 & $0 \%$ & \multirow[t]{3}{*}{$0.331 * *$} & \multirow[t]{3}{*}{$\mathrm{NS}$} \\
\hline & Improved & 17 & $26.1 \%$ & 19 & $21 \%$ & & \\
\hline & Resolved & 43 & $66.2 \%$ & 70 & $79 \%$ & & \\
\hline \multirow[t]{2}{*}{ Final outcome } & No change/improved & 22 & $33.8 \%$ & 19 & $21 \%$ & \multirow[t]{2}{*}{$0.243^{*}$} & \multirow[t]{2}{*}{$\mathrm{NS}$} \\
\hline & Resolved & 43 & $66.2 \%$ & 70 & $79 \%$ & & \\
\hline
\end{tabular}

*Chi-Square Tests. $\quad * *$ Fisher exact test

Table (11): Comparison between group 1 and 2 as regard systolic blood pressure at baseline and at follow up

\begin{tabular}{|c|c|c|c|c|c|c|}
\hline \multirow{2}{*}{\multicolumn{2}{|c|}{ Systolic Blood Pressure }} & Lap sleeve group & Lap MBG group & \multirow{3}{*}{$\begin{array}{c}\text { Test value• } \\
0.699 \bullet\end{array}$} & \multirow{3}{*}{$\begin{array}{c}\text { P-value } \\
0.489\end{array}$} & \multirow{3}{*}{$\begin{array}{l}\text { Sig. } \\
\text { NS }\end{array}$} \\
\hline & & No. $=68$ & No. $=71$ & & & \\
\hline \multirow{2}{*}{ Preoperative } & Mean \pm SD & $145.79 \pm 10.71$ & $143.33 \pm 11.44$ & & & \\
\hline & Range & $120-160$ & $120-170$ & & & \\
\hline \multirow[t]{2}{*}{1 month } & Mean \pm SD & $140.26 \pm 11.11$ & $138.10 \pm 9.28$ & \multirow[t]{2}{*}{$0.672 \bullet$} & \multirow[t]{2}{*}{0.506} & \multirow[t]{2}{*}{ NS } \\
\hline & Range & $120-160$ & $120-160$ & & & \\
\hline \multirow[t]{2}{*}{3 month } & Mean \pm SD & $135.53 \pm 9.70$ & $133.10 \pm 8.73$ & \multirow[t]{2}{*}{$0.834^{\bullet}$} & \multirow[t]{2}{*}{0.409} & \multirow[t]{2}{*}{ NS } \\
\hline & Range & $120-150$ & $120-150$ & & & \\
\hline \multirow[t]{2}{*}{6 month } & Mean \pm SD & $132.89 \pm 10.18$ & $129.76 \pm 7.98$ & \multirow[t]{2}{*}{$1.089 \bullet$} & \multirow[t]{2}{*}{0.283} & \multirow[t]{2}{*}{ NS } \\
\hline & Range & $120-150$ & $120-150$ & & & \\
\hline \multirow[t]{2}{*}{1 year } & Mean \pm SD & $130.79 \pm 9.32$ & $128.33 \pm 7.64$ & \multirow[t]{2}{*}{$0.915 \bullet$} & \multirow[t]{2}{*}{0.366} & \multirow[t]{2}{*}{ NS } \\
\hline & Range & $115-145$ & $120-150$ & & & \\
\hline $\begin{array}{l}\text { Total Decrease in } \\
\text { Systolic Blood } \\
\text { Pressure }\end{array}$ & & $15.00 \pm 7.45$ & $15.00 \pm 6.89$ & 0.00 & 1.00 & NS \\
\hline
\end{tabular}

NS: Non significant; S: Significant; HS: Highly significant

*:Chi-square test; $\bullet:$ Independent t-test

Table (12): Comparison between group 1 and 2 as regard diastolic blood pressure at baseline and at follow up.

\begin{tabular}{|c|c|c|c|c|c|c|}
\hline \multirow{2}{*}{\multicolumn{2}{|c|}{$\begin{array}{c}\text { Diastolic } \\
\text { Blood Pressure }\end{array}$}} & Lap sleeve group & Lap MGB group & \multirow{2}{*}{ Test value } & \multirow{2}{*}{ P-value } & \multirow{2}{*}{ Sig. } \\
\hline & & No. $=68$ & No. $=71$ & & & \\
\hline \multirow[t]{2}{*}{ Preoperative } & Mean \pm SD & $90.48 \pm 8.65$ & $86.32 \pm 8.95$ & \multirow[t]{2}{*}{$1.495^{\bullet}$} & \multirow[t]{2}{*}{0.143} & \multirow[t]{2}{*}{ NS } \\
\hline & Range & $80-110$ & $70-100$ & & & \\
\hline \multirow[t]{2}{*}{1 month } & Mean \pm SD & $86.19 \pm 5.90$ & $84.47 \pm 7.62$ & \multirow[t]{2}{*}{$0.801 \bullet$} & \multirow[t]{2}{*}{0.428} & \multirow[t]{2}{*}{$\mathrm{NS}$} \\
\hline & Range & $80-100$ & $70-100$ & & & \\
\hline \multirow[t]{2}{*}{3 months } & Mean \pm SD & $83.10 \pm 4.60$ & $81.05 \pm 5.91$ & \multirow[t]{2}{*}{$1.226^{\bullet}$} & \multirow[t]{2}{*}{0.228} & \multirow[t]{2}{*}{ NS } \\
\hline & Range & $80-90$ & $70-90$ & & & \\
\hline \multirow[t]{2}{*}{6 months } & Mean \pm SD & $81.67 \pm 5.08$ & $78.68 \pm 4.96$ & \multirow[t]{2}{*}{$1.875^{\bullet}$} & \multirow[t]{2}{*}{0.068} & \multirow[t]{2}{*}{ NS } \\
\hline & Range & $70-90$ & $70-85$ & & & \\
\hline \multirow[t]{2}{*}{1 year } & Mean \pm SD & $79.76 \pm 3.35$ & $76.58 \pm 6.25$ & \multirow[t]{2}{*}{$2.036^{\bullet}$} & \multirow[t]{2}{*}{0.049} & \multirow[t]{2}{*}{$\mathrm{S}$} \\
\hline & Range & $70-85$ & $60-85$ & & & \\
\hline
\end{tabular}

NS: Non significant; S: Significant; HS: Highly significant

*:Chi-square test; $\bullet$ : Independent t-test 
Table (13): The final outcome of hypertension between the 2 groups.

\begin{tabular}{|l|c|c|}
\hline & LSG $(\mathrm{n}=68)$ & $\begin{array}{c}\text { MGB } \\
(\mathrm{n}=71)\end{array}$ \\
\hline Resolved & $38(55.9 \%)$ & $42(60 \%)$ \\
\hline Improved & $20(29.4 \%)$ & $16(22 \%)$ \\
\hline No Change & $10(14.7 \%)$ & $13(18 \%)$ \\
\hline
\end{tabular}

Table (14): The final outcome of sleep apnea between the 2 groups:

\begin{tabular}{|l|c|c|}
\hline & $\begin{array}{c}\text { LSG } \\
(\mathrm{n}=73)\end{array}$ & $\begin{array}{c}\text { MGB } \\
(\mathrm{n}=69)\end{array}$ \\
\hline Resolved & $33(45 \%)$ & $35(51 \%)$ \\
\hline Improved & $25(35 \%)$ & $23(33 \%)$ \\
\hline No Change & $15(20 \%)$ & $11(16 \%)$ \\
\hline
\end{tabular}

\section{DISCUSSION}

Bariatric surgery has been proven to be a viable option for the treatment of severe obesity in comparison to conservative methods, resulting long lasting weight loss, improved quality of life, and resolution of obesity related co morbidities. It decreased overall mortality as well as morbidity in morbidly obese patients ${ }^{(5)}$.

Laparoscopic sleeve gastrectomy (LSG) has seen growth in popularity because of the perceived simplicity of the surgical technique, resolution of co-morbidities, and excellent weight loss outcomes. LSG has become widely considered as a primary restrictive bariatric procedure; LSG became the most commonly performed bariatric procedure (45.9\%) in 2014 According to the IFSO worldwide survey of $2014^{(6)}$.

The mini-gastric bypass (MGB) was introduced by Rutledge in 1997 and reported some years later. Since then, thousands of patients have been treated with this approach by several authors in different countries ${ }^{(7)}$.

Frequently, MGB is reported as an easier technique, to be preferred to other bariatric approaches, including both RYGB and $\mathrm{SG}$, for the results in both the short and long terms ${ }^{(7)}$.
Laparoscopic Sleeve Gastrectomy (LSG) is one of the principal bariatric procedure worldwide with excellent results for weight loss and reduction of co morbidities. Mini Gastric Bypass (MGB) has gained some popularity over years as a simple malabsorbtive bariatric procedure ${ }^{(8)}$.

The aim of our study was to compare between MGB and SG as regard weight loss, outcome of associated co-morbidities and complications. It is sample study which was done between December 2016 - December 2018, at Ain Shams university Hospitals, Cairo, Egypt.

This study included 200 patients, 100 patients in each group. The group age ranged between $22-55$ years with a mean \pm SD of $37.88 \pm 9.52$ years. Female patients represented the main population of this study $(60.5 \%)$. It is a common finding in the literature that women are undergoing bariatric surgery more than men ${ }^{(9)}$. We included in this study patients with $\mathrm{BMI} \geq$ 40 or $\mathrm{BMI}=35-39$ with one or more obesity-related co morbidities.

We used a 36 Fr bougie for sizing of the gastric sleeve. The American Society for Metabolic and Bariatric Surgery recommends now the use of a 34-40 Fr bougie to guide the stapling and maintain an adequate lumen of the gastric sleeve ${ }^{(10)}$. We used a $36 \mathrm{Fr}$ bougie for sizing of the gastric pouch in MGB. According to the IFSO Position Statement about Mini Gastric Bypass-One Anastomosis Gastric Bypass (MGB-OAGB) published in 2018 , the majority of studies used a $36 \mathrm{Fr}$ bougie; however, the bougie size varied from a $1 \mathrm{~cm}$ diameter nasogastric tube to a 42 French bougie $^{(11)}$.

The mean operative time for LSG in our study was 85 minutes ranging between 50-120 minutes, while in the MGB, it was 130 minutes ranging between 90-160 minutes. Statistically, the operative time for MGB was significantly longer than LSG. MGB 
consumes more time than LSG, due to mobilization of the bowel and sewing of the defects left after stapled gastrojejunostomy. The operative time for both techniques varies in the literature among different studies. Tucker et al. reported a mean operative time of 60 (58-190) minutes for LSG in primary cases $^{(12)}$. Young et al. analyzed the data of 5000 patients who underwent LSG using the American College of Surgeons National Surgical Quality Improvement Program database, and reported a mean operative time of 101 minutes for LSG in primary cases ${ }^{(13)}$.

\section{As regard complication:}

Complications in both procedures include hemorrhage, staple-line leak, stricture, obstruction, nutritional deficiencies, GERD, cholelithiasis, and weight-loss failure. LMGB has additional complications, in the form of marginal ulcer, anastomotic leakage, and chronic alkaline reflux. Compared with LMGB, LSG seems to have a smaller risk of complications, but the potential complications can be as severe as those associated with other techniques. The most feared complications after LSG and LMGB are leakage and hemorrhage ${ }^{(14)}$.

In our study hemorrhage was reported in 4 cases in SG group but it was reported in one case in MGB group and all cases were managed conservatively.

As regard postoperative leakage, it was reported in our study in two cases in SG group and was managed laparoscopic and UGE and one case in MGB group and had been converted to open.

As regard postoperative cholelithiasis, rapid weight loss is associated with the formation of cholesterol gallstones, within 6-12 months of the operation. The incidence was around $8.42 \%$ in the LSG group, and $12.7 \%$ in the LMGB group. The diagnosis is usually made by abdominal ultrasound during the follow-up period ${ }^{(15)}$. In our study, one case in SG group developed gall bladder stone and 3 cases in MGB group and 4 cases planned for cholecystectomy.

As regard mortality the incidence of mortality after LMGB ranged from 0 to $0.18 \%$; however, it was reported to be about $1.5 \%$ after LSG ${ }^{(16)}$. No mortality occurred in this study.

\section{As regard BMI loss:}

The mean BMI loss after one year in MGB $\left(19.67 \pm 7.17 \mathrm{~kg} / \mathrm{m}_{2}\right)$ was more than BMI loss in SG $(18.47 \pm 5.14 \mathrm{~kg} / \mathrm{m} 2)$ but this difference was statistically nonsignificant. In comparing to the study of Milone and his colleagues (to compare between SG and MGB after one year) in which, SG and MGB were associated with changes in BMI $(20.33 \pm 4.48 \%$ vs $19.19 \pm$ $4.42 \%)$ and also, the difference between them was statistically non-significant ( $P$ value $=0.931)^{(17)}$.

Wang and his colleagues published their results on 423 consecutive patients (87 males and 336 females) underwent laparoscopic MGB for morbid obesity. The BMI decreased from 44.2 to $35.1,31.9$, and 29.2, at 3, 6 and 12 months respectively with total BMI loss after one year was 15 $\mathrm{kg} / \mathrm{m} 2^{(18)}$. The mean BMI loss in our study in MGB group was better than Wang study.

In the study done by Musella and his colleagues, after SG, BMI decreased from basal 47.9 to 32.6 after one year with total BMI loss after one year was $15.3 \mathrm{~kg} / \mathrm{m} 2$ (less than in our study) while in MGB group, BMI decreased from basal 50.8 to 29.2 after one year with total BMI loss was $21.6 \mathrm{~kg} / \mathrm{m} 2$ (better than in our study) $^{(7)}$.

\section{As regard diabetes, HTN and sleep apnea remission effect:}

MGB has a better effect than SG in diabetes remission detected by that the mean FBS drop after one year in MGB (37.80 \pm $6.41 \mathrm{mg} / \mathrm{dl})$ was more than after SG $(29.93 \pm$ $12.84 \mathrm{mg} / \mathrm{dl}$ ) and this difference of drop was 
highly statistically significant ( $\mathrm{p}$ value $<0.004$ ).

The mean HBAlc drop after one year in MGB $(2.33 \pm 0.48 \%)$ was more than in SG $(2.01 \pm 0.59 \%)$ and this difference of drop was statistically significant ( $p$ value $<0.024$ ).

So, Complete resolution of diabetes occurred in MGB cases in 79\% compared to $66.7 \%$ in SG cases at 12 months and cases with no remission in D.M was $0 \%$ in MGB and $7.7 \%$ with SG.

The universal published data shows similar results to our study. A retrospective study by Lee and his colleagues including 62 T2DM obese patients underwent gastrointestinal surgery (LMGB and LSG). After one year the result was remission of T2DM achieved in $45(72.5 \%)$ patients after these different operations. A comparison among three different operative methods revealed remission rate of T2DM was achieved in $84.8 \%, 58.8 \%$ and $58.3 \%$ of patients for LMGB, LAGB and LSG, respectively. LMGB had the best remission effect on T2DM $(85 \%)$ at 1 year after surgery compared with LAGB and LSG. Among the different operative methods, waist circumference and C-peptide levels were determined to be significant predictors for the remission of T2DM in obese patients ${ }^{(5)}$. The result of our study agreed with this study as regard that both operation are effective in diabetes remission but MGB has better effect than SG.

Another study done by Milone and his colleague. The patients were split into two groups according to the surgical intervention performed, sleeve gastrectomy and minigastric bypass. A total of 53 subjects who underwent sleeve gastrectomy or mini-gastric bypass for obesity and diabetes were screened for the inclusion in this study. Of these, 4 subjects were excluded because of surgical complications, 7 subjects were omitted because young surgeons conducted the operations and 11 subjects were removed because of the lack of follow-up. Thirty one obese patients were recruited for this study. A total of 15 subjects underwent SG (48.4\%), and 16 underwent MGB (51.6\%).The prevalence of diabetes remission was gradually increased following surgery, regardless of the type, specifically, at 3 months post-surgical intervention, diabetes remission was reported by 18 subjects $(53.3 \%$ in SG vs $62.5 \%$ in $\mathrm{MGB}, \mathrm{P}=0.722)$. Similar results were confirmed at the 6 months follow-up (53.3\% for $\mathrm{SG}$ vs $68.8 \%$ for $\mathrm{MGB}, \mathrm{P}=$ 0.473 ).At the 12 months follow-up, $66.7 \%$ of subjects who underwent SG achieved diabetes remission vs $87.5 \%$ of those who underwent MGB ( $\mathrm{P}=0.220)$. High preoperative HBA1c was determined to be a negative predictor of diabetes remission at 12 months while there was significant correlation between percent of BMI loss and diabetes remission in both operation ${ }^{(17)}$. The result of our study agreed with this study as regard MGB has better and faster effect on diabetes remission than SG although the effect of both operation at 6 months are better in Milone study than our study.

The study also revealed positive correlation between BMI loss and diabetes remission but statistically non significant (may indicating presence of other more important mechanisms of postoperative diabetes resolution rather than weight loss).

In the retrospective study by Lee and his colleagues to compare between different gastrointestinal surgery (LMGB and LSG) among the different operative methods, waist circumference and C-peptide levels were determined to be significant predictors for the remission of T2DM in obese patients ${ }^{(5)}$.

As regard HTN in our study, in group 1 (SG) resolution of hypertension was $55.9 \%$ (38 cases), improvement was 29.4\% (20 cases) and no change in hypertension status was $14.7 \%$ (10cases), in group 2 (MGB) resolution of hypertension was $60 \%$ (42cases), improvement was 22\% (16 cases) 
and no change in hypertension status was $18 \%$ (13 cases).

As regard sleep apnea in our study, in group 1 (LSG) resolution of sleep apnea was $45 \%$ (33 cases), Improvement was 35\% (25 cases) and no change in sleep apnea was $20 \%$ (15 cases), In group 2 (LMGB) resolution of sleep apnea was 51\% (35 cases), Improvement was 33\% (23 cases) and no change in sleep apnea was 16\% (11 cases), with comparing the 2 groups.

Approximately $80 \%$ of obese adults have at least one, and $40 \%$ have two, or more associated diseases such as T2DM, hypertension, cardiovascular disease, cancers, dyslipidemia and/or insulin resistance. Weight loss is associated within improvement in fasting glucose, insulin resistance and dyslipidemia. Several surgical studies have demonstrated the safety and efficacy of bariatric surgery, especially in terms of reduction in comorbidities over time ${ }^{(19)}$.

Finally our study suggests that bariatric surgery (mainly SG and MGB which are the most common bariatric operations nowadays) are considered the best treatment of diabetes type 2 in obese patients and MGB is superior to $\mathrm{SG}$ in diabetes remission.

Both procedures are also associated with improvement of the blood pressure and the sleep apnea owing to weight loss.

\section{Conclusion:}

It is reasonable to say that both LSG and MGB achieve similar weight loss mean and resolution of co-morbidities at 1 year.

\section{REFERENCES}

1. Schauer PR, Kashyap SR, Wolski K, Brethauer SA, Kirwan JP, Pothier CE, Thomas S, Abood B, Nissen SE and Bhatt DL (2012): Bariatric surgery versus intensive medical therapy in obese patients with diabetes. N Engl J Med. 366:15671576.
2. Messiah SE, Arheart K, Hlaing W, Natale R, Lipshultz SE and Miller TL (2013): Body mass index, waist circumference, and cardiovascular disease risk factors among preschool-age children in the United States. Obesity. doi:10L1038/oby.2013.353. 2013.

3. Shabbir A and Teh JL A. New Emerging procedure - Sleeve Gastrectomy. In: Essentials and Controversies in Bariatric Surgery. InTech; 2014.

4. Reis CE, Alvarez-Leite JI, Bressan J and Alfenas RC (2012): Role of bariatricmetabolic surgery in the treatment of obese type 2 diabetes with body mass index $<35$ $\mathrm{kg} / \mathrm{m}^{2}$ : a literature review. Diabetes Technol Ther. 14:365-372.

5. Lee WJ, Ser KH and Lee YC (2012): Laparoscopic Roux-en-Y vs. Mini gastric bypass for the treatment of morbid obesity: a 10-year experience. Obes Surg; 22:182734.

6. Angrisani L, Santonicola A, Iovino $\mathrm{P}$, Vitiello A, Zundel N, Buchwald $\mathrm{H}$ and Scopinaro N (2017): Bariatric Surgery and Endoluminal Procedures: IFSO Worldwide Survey 2014. Obes Surg. 27(9):2279-2289.

7. Musella M, Susa A, Greco F, De Luca M, Manno E, Di Stefano C, Milone M, Bonfanti R, Segato G, Antonino A, and Piazza L (2014): Surg Endosc 28:156-163.

8. Kowalewski PK, Olszewski R, Waledziak MS, Janik MR, Kwiatkowski A and Galazka-Swiderek N (2018): Long-term outcomes of laparoscopic sleeve gastrectomy-a singlecenter, retrospective study. Obes Surg. 28:130-134

9. Miller-Matero LR, Tobin ET, Clark S, Eshelman A and Genaw J (2016): Pursuing bariatric surgery in an urban area: gender and racial disparities and risk for psychiatric symptoms. Obes Res Clin Pract. 10(1):5662.

10. Zundel N, Hernandez JD and Gagner M (2015): Laparoscopic sleeve gastrectomy: technique and outcomes. The ASMBS textbook of bariatric surgery: Springer. p. 205-10. 
11. De Luca M, Tie T, Ooi G, Higa K, Himpens J and Carbajo MA (2018): Mini Gastric Bypass-One Anastomosis Gastric Bypass (MGB-OAGB)-IFSO Position Statement. Obesity surgery. 28(5):1188-206.

12. Tucker O, Szomstein $\mathrm{S}$ and Rosenthal $\mathrm{R}$ (2008): Indications for sleeve gastrectomy as a primary procedure for weight loss in the morbidly obese. Journal of Gastrointestinal Surgery. 12(4):662-7.

13. Young MT, Gebhart A, Phelan MJ and Nguyen NT (2015): Use and outcomes of laparoscopic sleeve gastrectomy vs laparoscopic gastric bypass: analysis of the American College of Surgeons NSQIP. J Am Coll Surg. 220(5):880-5.

14. Mahawar KK, Carr WR, Balupuri $S$ and Small PK (2014): Controversy surrounding minigastric bypass. Obesity surgery. 24(2):324-33.
15. Mishra T, Lakshmi KK and Peddi KK (2016): Prevalence of cholelithiasis and choledocholithiasis in morbidly obese South Indian patients and the further development of biliary calculus disease after sleeve gastrectomy, gastric bypass and mini gastric bypass. Obes Surg. 26:2411-2417.

16. Victorzon M (2015): Single-anastomosis gastric bypass: better, faster, and safer? Scand J Surg. 104:48-53.

17. Milone M, Lupoli R and Maietta P (2015): Lipid profile changes in patients undergoing bariatric surgery: a comparative study between sleeve gastrectomy and minigastric bypass. Int J Surg. 14:28-32.

18. Wang $\mathrm{YC}$, McPherson $\mathrm{K}$, Marsh $\mathrm{T}$, Gortmaker SL, Brown M (211): Health and economic burden of the projected obesity trends in the USA and the UK. Lancet.:815-825.

19. Perry Y, Courcoulas AP, and Fernando HC (2008): Laparoscopic Roux-en-Y gastric bypass for recalcitrant gastroesophageal reflux disease in morbidly obese patients. JSLS; 8:19-23. 


\section{Osama Ali EIAtrash, et al.,}

نتائج عمليتى تكميم المعدة بالمنظار وتحويل مسار المعدة المصغر بالمنظار فى المرضى المصريين الأين يعانون من مرض المنطئة السمنة المفرطة

$$
\begin{aligned}
& \text { أسامة على الأطرش، وليد ابراهيم عبد الحميد، محمد عبد المنعم مرزوق، } \\
& \text { محمد عبد الستار عبد الحميد، أحمد فاروق عبد الحبد الحفيظ }
\end{aligned}
$$

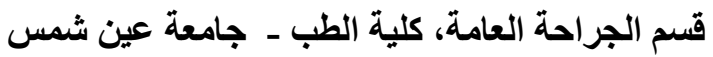

خلفية: تعتبر الأمر اض المزمنة من اهم اسباب الوفاة وتعتبر السمنة المفرطة سبب رئيسي لتلك الأمر اض و التي تهدد الصحة العالمية.

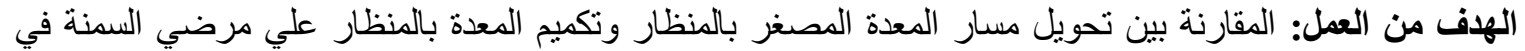

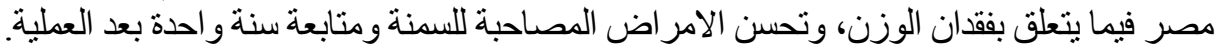

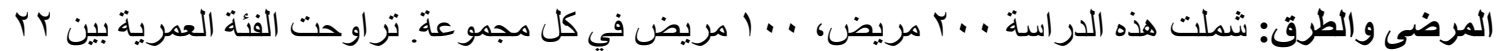

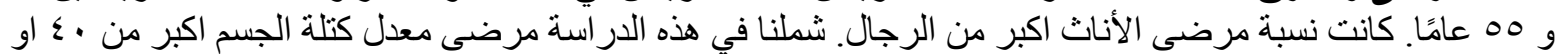

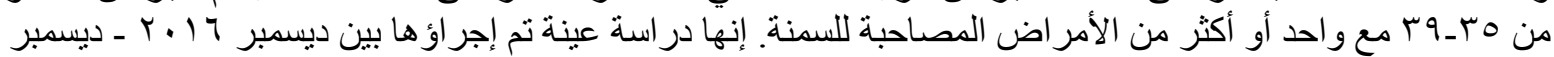

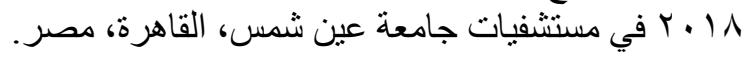

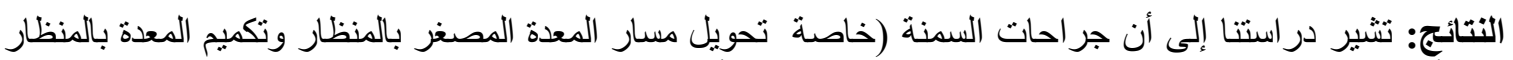

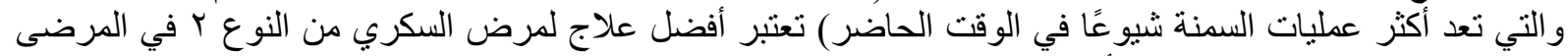

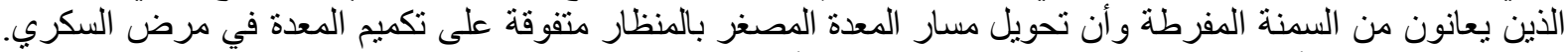

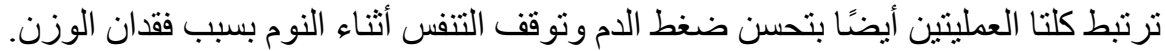

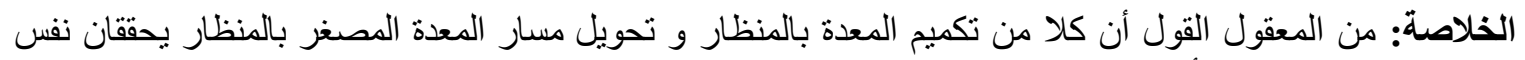
معدل إنقاص الوزن وتحسن الأمر اض المصاحبة للسمنة ومتابعة سنة واحدة بعد العملية. كلمات البحث: السكر التر اكمي، تحويل مسار المعدة المصغر بالمنظار ، تكميم المعدة بالمنظار 